\title{
Using Composition Trees to Validate an Entry Profile of Software Engineering Lifecycle Profiles for Very Small Entities (VSEs)
}

\author{
Lian Wen ${ }^{1,2}$, Terry Rout ${ }^{1}$, \\ ${ }^{1}$ Institute for Integrated and Intelligent Systems, Griffith University \\ ${ }^{2}$ School of Information and Communication Technology, Griffith University \\ 170 Kessels Rd, Q1d 4111, Australia \\ \{l.wen, t.rout\}@griffith.edu.au
}

\begin{abstract}
ISO/IEC TR 29110-5-1 provides a Software Engineering life cycle reference model for Very Small Entities on small software projects (less than 6 people month). This paper uses Composition Trees (CT) as a formal notation to model part of this process, and compares it with its counterpart process (which is also modeled in a CT) from ISO/IEC 12207. The outcome of the comparison is a Comparison Composition Tree (CCT). This CCT shows clearly the similarity and difference between the VSE Entry Profile and ISC12207. This information may help people to validate and understand the VSE Profile. This paper also proposes this approach can be used as a general approach for people to develop, study, and implement software processes.
\end{abstract}

Keywords: Composition Trees, Very Small Entity, Software Process,

\section{Introduction}

Model-based process improvement has been widely applied to support the design, implementation and improvement of processes for the development of complex products [27], especially in the domain of systems and software engineering. Significant benefits have been shown to derive from the application of model-based improvement [8], and it is common for acquirers to require demonstration of some degree of implementation of effective processes as part of an evaluation of supplier capability. A wide range of techniques have been applied for the definition and specification of industrial processes, having widely differing degrees of formality.

In the domains of software and system engineering, a range of Standards and related products have been developed specifying processes to be employed in the product life cycle - see, for example, ISO/IEC 12207:2008 [10], ISO/IEC 15288:2008 [11] and the Capability Maturity Model Integration suite [3]. These Standards are predominantly descriptive and prescriptive in nature. We have highlighted in a previous paper [25] the problems that can arise in comparing similar processes defined in different contexts, in some cases using different modeling approaches. 
In seeking a solution to these problems in process modeling, similar problems can be identified with the requirement specifications for software systems. Ambiguous language, incomplete descriptions, repetition and redundancies in the way specifications are expressed inevitably lead to sub-optimal project outcomes (systems that do not meet the user's needs). Behavior Engineering [1, 4] successfully addresses the problems faced by software developers seeking to translate a set of user requirements into a complete and consistent requirements specification.

Behavior Engineering uses a formally-grounded graphical notation with the capability to represent a wide range of system behaviors in unambiguous terms. Its strength is its ability to accommodate complexity and detail, ease of use, and in particular for this project its ability to expose defects.

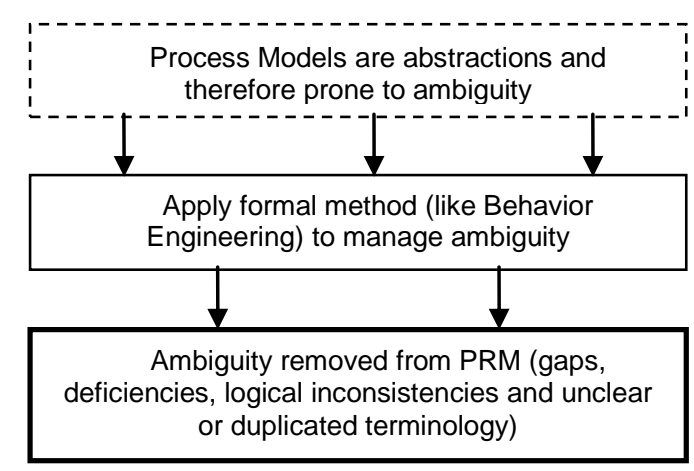

Fig. 1 - Use of formal method to remove ambiguity from abstract model

Previous research indicates that BE notations can be useful verification tools for process modeling [22]. In our previous paper we refined this concept by proposing a detailed scheme to model a software process based on its purpose and process outcomes in a Composition Tree (CT) [6], which is one of the key parts of the BE notations. The graphic version of a process model is more intuitive, less ambiguous and easier to verify than the original natural language described process.

The application of Behavior Engineering to process modeling offers significant benefits, in terms of clarifying process descriptions and specifically in helping to establish process-related risk associated with process tailoring. In the current investigations, we report on the application of the technique to analysis of tailoring of process models for Very Small Enterprises.

\section{Lifecycle Profiles for Very Small Entities}

Very Small Enterprises (VSEs) are defined as those having 25 or fewer personnel. It has been widely recognized that enterprises in this class make a very significant contribution to the IT industry [18]. However, it is also acknowledged that most VSEs do not find the process models typically available and useful for their purposes, primarily because of associated high bureaucratic overheads. Actions have been taken to develop specific models more suitable for the use of VSEs, generally by 
tailoring existing approaches; the most widely recognized of these is the development of the ISO/IEC 29110 [13] series of Standards.

ISO/IEC 29110 specifies process models for VSEs as "standard lifecycle profiles", following the approach described in ISO/IEC 10000-1 [9]. The Profiles are designed for use both for internal, model-based improvement within the VSE, and for use in the assessment of overall organizational capability [16]. ISO/IEC 29110 provides for the definition of a range of profiles, tailored from processes specified in the source Standard, ISO/IEC 12207 [10], and designed for enterprises engaged in different classes of development.
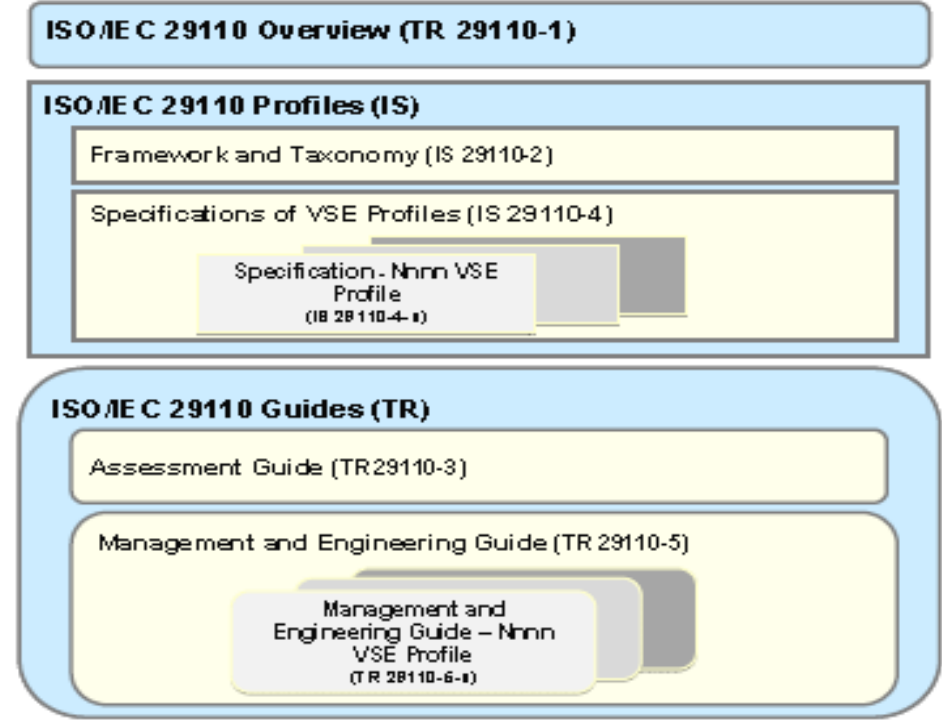

Fig. 2 - ISO/IEC 29110 set of Documents [13]

The document set for ISO/IEC 29110 is based around a three-tiered structure, shown in Fig. 2. At the top level, there is an overview of the approach [13]. Normative elements of the standard suite comprise a Framework and Taxonomy for specifying profiles [14], and an envisaged set of Profile Specifications [15]; these specify the structure and content of profiles to be developed for specified classes of users. The detailed Profiles are set out in the Management and Engineering Guides [17]; these are implementations of tailored process descriptions, based on the source Standards, and employing the relevant Specifications. Multiple different profiles are to be established for different classes of VSEs and projects; in the initial phase of development, a set of "Generic Profiles" have been developed and published.

In the profile specifications, ISO/IEC 29110 follows the approach defined in ISO/IEC 24774:2007 - Software and systems engineering -- Life cycle management -Guidelines for process description [12], which outlines a standard format for any process reference model, including those intended for process implementation and process assessment. This general purpose standard outlines the elements used to describe a process; title, purpose statement, outcomes, activities and tasks.

- The title conveys the scope of the process as a whole, expressed as a short noun phrase that summarize the scope of the process, identify the principal 
concern of the process, and distinguishes it from other processes within the scope of a process model.

- The purpose describes the goal of performing the process. It is expressed as a high level goal for performing the process, preferably stated in a single sentence. The implementation of the process should provide measurable, tangible benefits to the stakeholders through the expected outcomes

- The outcomes express the observable results expected from the successful performance of the process. Outcomes are expressed in terms of a positive, observable objective or benefit. The list of outcomes associated with a process shall be prefaced by the text, "As a result of successful implementation of this process:'. The outcomes should be no longer than two lines of text, about twenty words. The number of outcomes for a process should fall within the range 3 to 7 . Outcomes should express a single result. The use of the word 'and' or 'and/or' to conjoin clauses should be avoided.

- The activities are a list of actions that may be used to achieve the outcomes. Each activity may be further elaborated as a grouping of related lower level actions;

- The tasks are specific actions that may be performed to achieve an activity. Multiple related tasks are often grouped within an activity.

In ISO/IEC 29110-5-1 [17], a Profile is specified for VSEs undertaking software development of a generic class, tailored from ISO/IEC 12207. The Profile contains two identified Processes, each comprising a set of high-level tasks; the tasks and processes are derived by tailoring and assembling process components from ISO/IEC 12207 [10]. The impact of the tailoring is not immediately obvious, and the implications of the application of these profiles in an organization are not clear. For this reason, we have undertaken an analysis of the available profiles, using Behavior Engineering approach, and applied Composition Trees to specifically determine the impact of the tailoring.

\section{Using Composition Trees to Model and Compare Software Processes}

\subsection{Composition Trees}

A Composition Tree (CT) is originally used to describe the composition of a component based software intensive system [6]. It provides useful summary information including states, attributes and relationships about the system and other entities under the system. The CT notation is then adapted to model software processes [25] and manufacture processes [26].

Similar to the way of constructing a Behavior Tree from the functional requirements [5], A Composition Tree can also be constructed through translating the individual functional requirements one by one. In this sub section, we use a small example to show what a CT looks like (in Fig. 3). Detailed and formal introduction of CT can be found somewhere else $[1,25]$ 


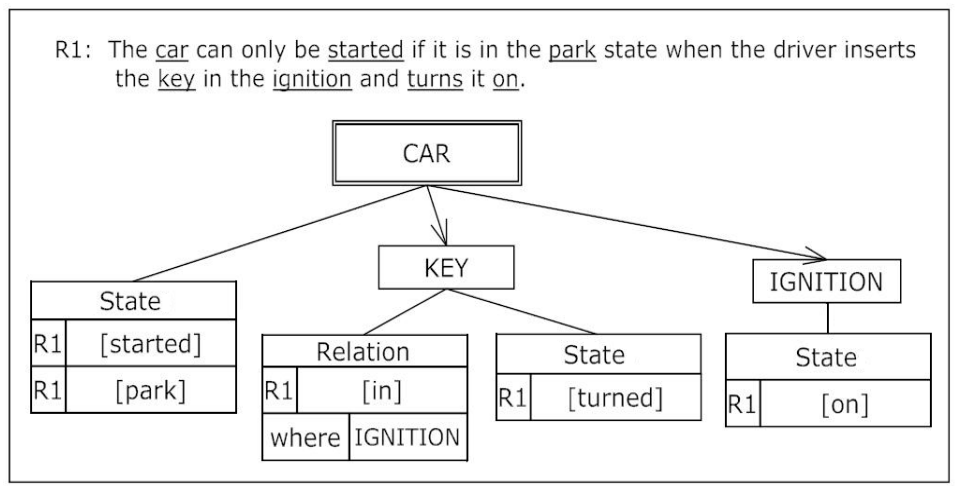

Fig. 3 - The Composition Tree (CT) generated from translating requirement 1 (R1). Within the CAR system, there are two components: KEY and IGNITION. The CAR has two possible states: [started] and [park], while the KEY has one state of [turned] and one state [on] for the component IGNITION. There is a relation [in] between the KEY and the IGNITION.

The process to translate all the requirements into one CT has following advantages:

- All information is integrated together so it is easy to indentify the requirement defects. For example, the incompleteness and inconsistency in the original requirements.

- A CT arranges the information about one component in one place. It will be easier for people to design and implement the components than the original requirements with the information of one component may be scattered all around the requirements.

- The more specific graphic notation is less ambiguous than the more flexible natural language.

- A CT removes the entire alias so it will use a consistent vocabulary for the system.

The advantages of using CT in Software and System Engineering has been demonstrated elsewhere [6], further details are not provided in this paper.

\subsection{Using Composition Trees to Model Software Processes}

According to ISO/IEC 24774:2007 [12], the standard elements to describe a process include the title, the purpose, outcomes, activities and tasks. Apart from the title, which is only the name of a process, purpose and outcomes are more static elements, so they may be more suitable to be modeled by composition trees.

Fig. 4 is the CT for the purpose of the Configuration Management Process defined in ISO/IEC 12207. The CT for the whole process and the detailed translation steps can be found in [25]. 


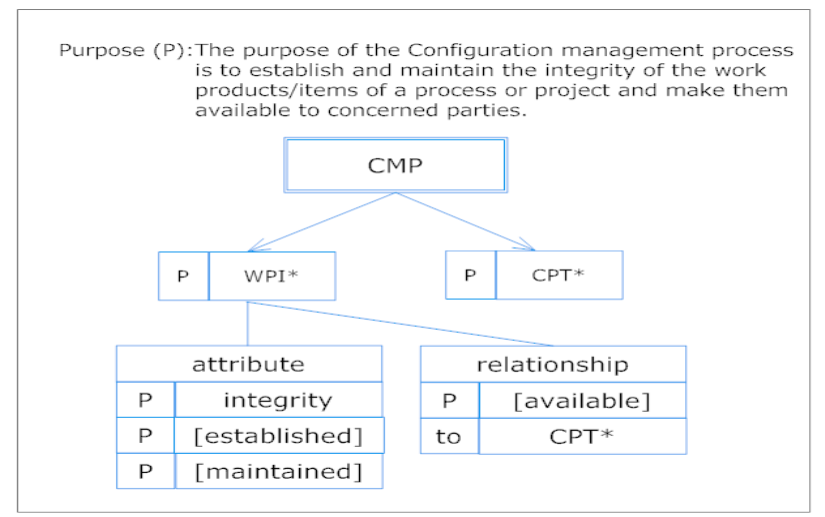

Fig. 4 - The Composition Tree constructed from the purpose of the Configuration Management Process.

CMP stands for Configuration Management Process; WPI means Work Product/Item, CPT means Concerned Parts. "*” means this component may have multiple instances

\subsection{Comparing Composition Trees}

One special advantage to model software processes in composition trees is that we can define formal algorithm to compare the two composition trees. Therefore, it provides a simple method to identify the similarity and difference between two related processes. While it could be more challenge if we try to compare two processes simply based on their natural language descriptions.

The comparison implements a label matching tree merging algorithm which has been used for comparing different versions of Behavior Trees [24].

A critical task in tree merging algorithm is to identify the matching nodes. For CTs, the way to identify the same nodes is based on the name of the component and its state, etc. Therefore, before applying the merging algorithm, the first step is to identify the same component and/or the same state which may be called by different names in the two compared trees and to establish a mapping between them.

The second step is to compare and merge the two trees. To simplify the discussion, we may call the first tree as the old tree and the second tree as the new tree. In this way, the comparison procedure will create a merged tree that is called a Comparison Composition Tree (CCT). A CCT shows all the information of both trees and also highlights the difference in an easy to read way. To achieve this purpose, a display style convention is used in this paper as in Fig. 5.

Under this display style convention, in a CCT, a piece of information which exists in both the old tree and the new tree is called unchanged and will be drawn in normal style; a piece of information if only exists in the old tree will be called old and will be drawn in dotted lines; a piece of information if only exists in the new tree will be called new and will be drawn in bolded lines. 


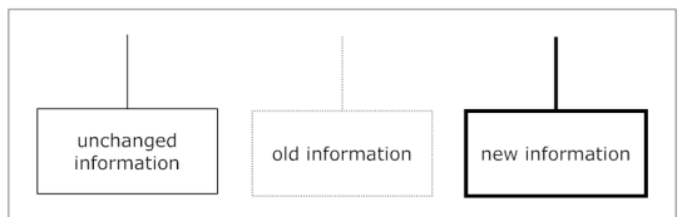

Fig. 5 - The display style convention for a CCT

\section{Comparing the VSE Entry Profile with ISO/IEC 12207}

In this section, we compare one of the process objectives defined in the VSE Entry Profile [17] with their more comprehensive counterparts defined in ISO/IEC 12207 [10] through their CT modeling. Because the comparison models show the similarities and differences in a clear, simple, and easy to read way, they can be useful for people to validate the profile for its usefulness regarding to the VSE.

The Entry Profile of VSE defines only two processes: Project Management (PM) process and Software Implementation (SI) process, while ISO/IEC 12207:2008 has defined 43 processes. Each process in VSE Entry Profile has a number of objectives with each objective includes certain elements which are covered by one or more processes defined in ISO/IEC 12207.

In this paper, we firstly investigate the object $1(\mathrm{O} 1)$ in the PM process from the VSE Entry Profile. PM.O1 mainly focuses on the project plan that is mostly defined in the Project Planning Process in ISO/IEC 12207.

According to ISO/IEC 29110-5-1 [17], PM.O1 is:

The Project Plan for the execution of the project is developed according to the Statement of Work and reviewed and accepted by the Customer. The tasks and resources necessary to complete the work are sized and estimated.

The CT of PM.O1 is shown in Fig. 6:

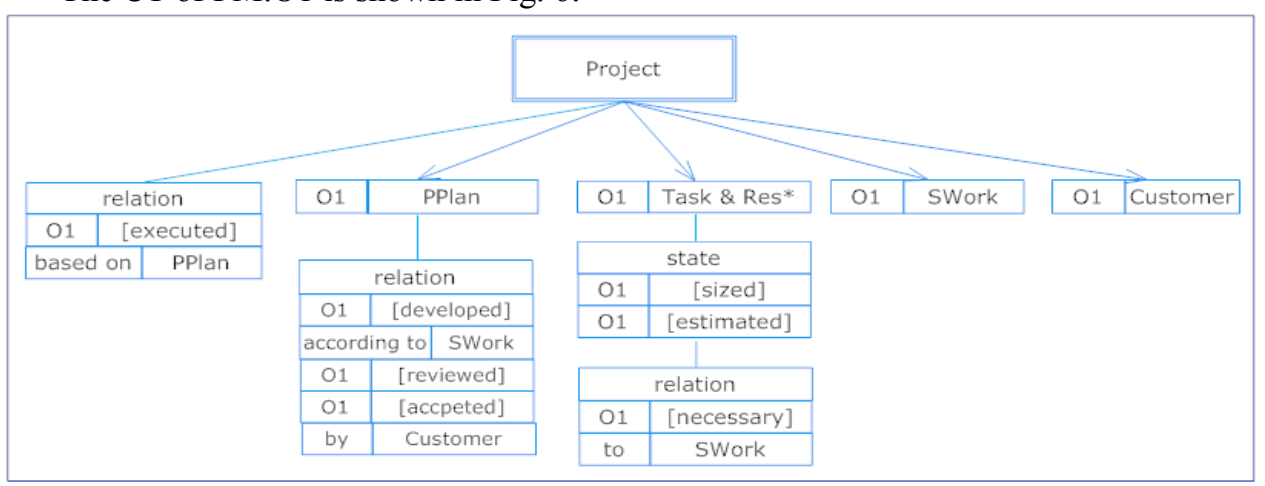

Fig. 6 - The Composition Tree (CT) modeling of the first object in the Project Management process in the VSE Entry Profile

PPlan: Project Plan

SWork: Statement of Work
Task \& Res: Task and resource.

O1: Object one of the project management process 
Fig. 6 shows that: the Project is executed based on the Project Plan (PPlan); the Project includes a Project Plan, multiple Tasks and Resources (the '*' sign means plural), Statement of Work (SWork) and a Customer; the Project Plan is developed according to the Statement of Work (SWork), and the Project Plan is reviewed and accepted by the Customer; the Tasks and Resources are sized and estimated, they are also necessary to the Statement of Work.

Compared the graphic notation with the natural language description of PM.O1, it is easier to identify the relationship between different concepts is the CT notation.

Secondly, we identify that the counterpart process in ISO/IEC 12207 is the Project Planning Process. The purpose and outcomes of the Project Planning Process are:

Purpose: The purpose of the Project Planning Process is to produce and communicate effective and workable project plans.

This process determines the scope of the project management and technical activities, identifies process outputs, project tasks and deliverables, establishes schedules for project task conduct, including achievement criteria, and required resources to accomplish project tasks.

\section{Outcomes:}

a) the scope of the work for the project is defined;

b) the feasibility of achieving the goals of the project with available resources and constraints are evaluated;

c) the tasks and resources necessary to complete the work are sized and estimated;

d) interfaces between elements in the project, and with other project and organizational units, are identified;

e) plans for the execution of the project are developed; and

f) plans for the execution of the project are activated.

We draw the Composition Tree model of the Project Planning Process in Fig. 7. This model is built based on the process purpose and the process outcomes. This composition tree uses the following abbreviations:

Task \& Res: task and resources; OUnit: Other project \& organizational unit.

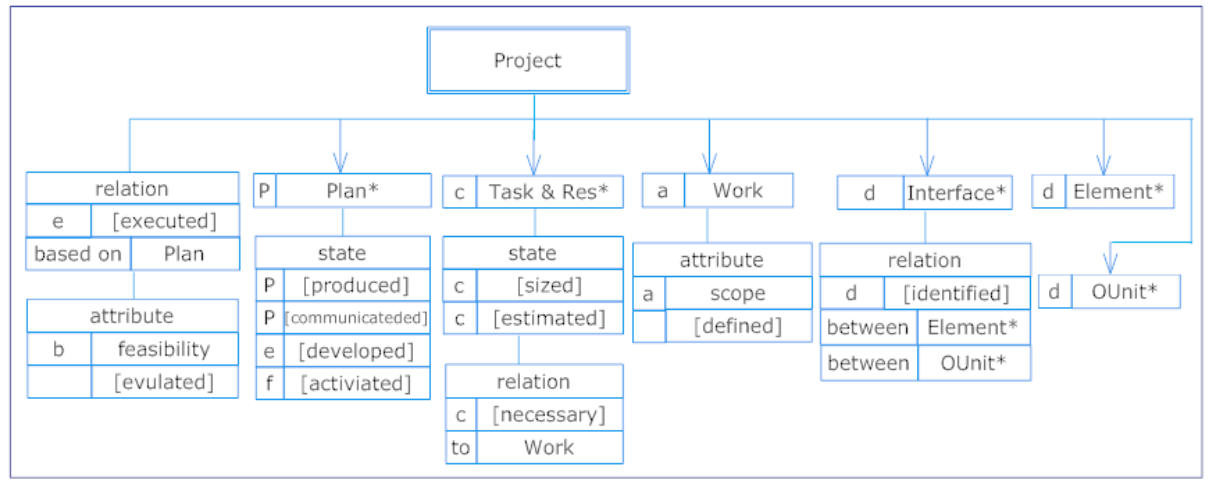

Fig. 7 - The Composition Tree for the Project Planning Process in ISO/IEC 12207. This model is based on the process purpose and process outcomes. 
Finally, we compare the two composition trees based on the algorithm introduced in the previous section. Before the comparison, we need to identify the mapping terms between the two composition trees. The mapping terms are listed in Table 1.

Table 1 - The mapping terms between PM.O1 and the Project Planning Process.

\begin{tabular}{|l|l|l|l|}
\hline$\#$ & PM.O1 & Project Planning Process & \multicolumn{1}{|c|}{ Comments } \\
\hline 1 & PPlan & Plan* & $\begin{array}{l}\text { For an Entry Profile in VSE, a simple } \\
\text { Project Pan is sufficient; while for a } \\
\text { more general project, multiple plans } \\
\text { would be required. }\end{array}$ \\
\hline 2 & SWork & Work & $\begin{array}{l}\text { For an Entry Profile, the work is } \\
\text { materialized as a Statement of Work. }\end{array}$ \\
\hline
\end{tabular}

The composition tree for the Project Planning Process in ISO/IEC 12207 is called the old tree (shown in Fig. 7); the composition tree for PM.O1 is called the new tree (shown in Fig. 6). Then we apply the tree merging algorithm to create the Comparison Composition Tree (CCT) as in Fig. 8.

In Fig. 8, the information exists in both ISO/IEC 12207 and the VSE is displayed in the normal style, the information is called unchanged. The information exists only in ISO/IEC 12207 is called old, that means this information has been removed from the VSE, and it is displayed in gray boxes. The information exists only in the VSE is called new, and it is displayed in bold boxes.

The CCT highlights the difference between the PM.O1 of VSE Entry Profile and its counterpart process defined in ISO/IEC 12207. The difference helps people to understand the rationality behind the design of the Profile. In next section, we will go through the details of CCT and illustrate this point.

\section{Discussion}

In the previous section, we have created the CCT of the ISO/IEC 12207 Project Planning Process and the PM.O1 of the VSE Entry Profile in Fig. 8. In this section, we will go through the details of the CCT to examine what information can be directly retrieved from the $\mathrm{CCT}$ and how it could be used to justify the rationality of the VSE Entry Profile.

To read the CCT, we go through all branches one by one from the leftmost.

The leftmost branch shows that for both ISO/IEC 12207 and the VSE Profile, the Project should be executed based on plan. However, the VSE Profile does not mention that the feasibility of the project should be evaluated. The reason for this point being removed from the VSE Profile is that the VSE Profile is designed for small project team (up to 25 people in the organization) and with small projects. The feasibility of the project is easy to be evaluated and it should have been evaluated earlier, so it is not necessary to be a formal part of the planning process. 


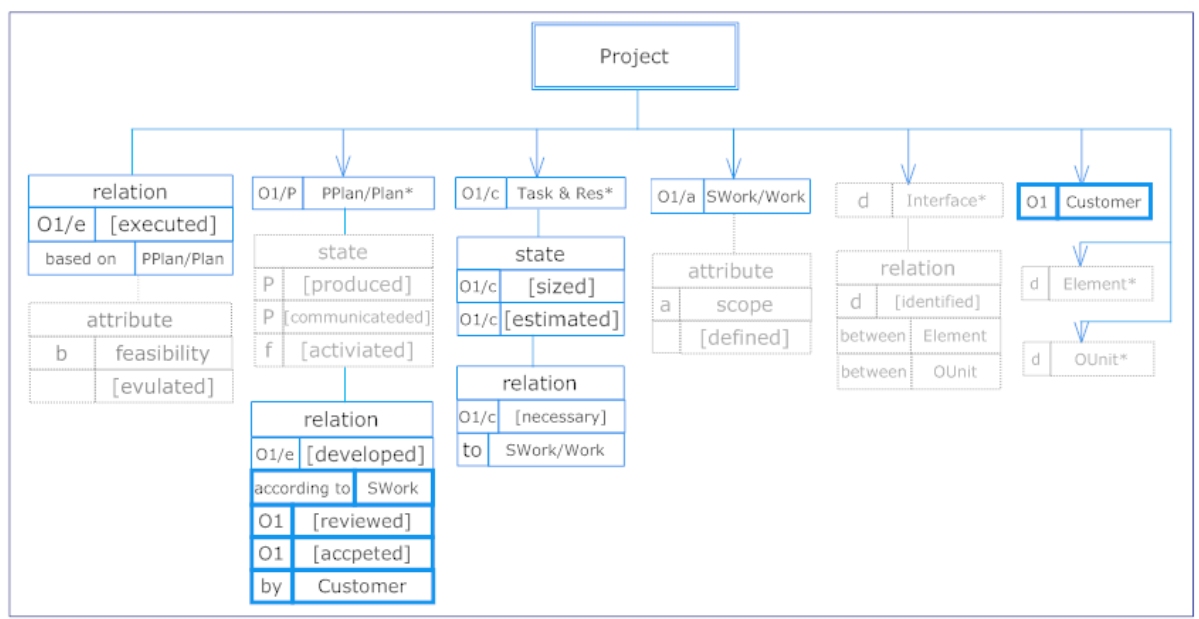

Fig. 8 - The Comparison Composition Tree (CCT) generated from merging the composition tree of the Project Planning Process in ISO/IEC 12207 and the PM.O1 from the VSE Entry Profile.

The second branch in the CCT is about the plan document(s). An obvious difference is that the VSE Profile only has one Project Plan while ISO/IEC 12207 may have multiple plans for the project. This difference is easy to understand as the VSE Profile handles very small projects, one project plan should be efficient.

The second difference in the second branch is that the VSE Profile doesn't mention that the Project Plan should be produced, communicated and activated. Of course, the plan should be created, communicated and activated even for small projects. The reason for it is not mentioned in the VSE Profile is these requirements are too obvious to be formally addressed. However, for large projects which involve many stakeholders and many different kinds of plans, the requirements should be explicitly addressed.

The third difference in the second branch is that the VSE Entry Profile mentions that the Project Plan should be reviewed and accepted by the Customer, while this point is not mentioned in the Project Planning Process in ISO/IEC 12207. The reason is that ISO/IEC 12207 builds a much large and complex process groups to handle the procedures to create, verify and accept any formal documents, therefore, it doesn't need to repeat it in the Project Planning Process for this point.

There is no much difference in the third branch.

The first interesting point in the fourth branch is that the VSE Entry Profile uses the Statement of Work while ISO/IEC 12207 uses a more general term Work. The difference can be explained that for a small project, the work is already well expressed in the Statement of Work. However, for large projects, it may not have a formal statement of work at the planning stage, so it use a more general term Work. The second point is that as ISO/IEC 12207 uses a more general term Work, it also need to define the scope of the work in the planning process, but this is not required if we already have a Statement of Work in the VSE Profile.

The fifth branch only exists in ISO/IEC 12207. The branch expresses that the interfaces between different Elements and other Project and Organization Units are 
identified. As the VSE Profile only deals with small projects; they are usually small and simple, so the identification of the interfaces between different internal and/or external parts is usually not an issue.

The last branch in the CCT shows that Customer appears only in the VSE Profile while the Elements and OUnits only appear in the ISO/IEC 12207 Project Planning Process. The reasons have already been discussed in the previous paragraphs.

In this section, we go through the details of the CCT. We find that the CCT uses a clear and easy to read way to highlight the difference and similarity between the PM.O1 and the Project Planning Process. This information helps to understand, reason and validate the VSE Profile

\section{Conclusions and further research topics}

This paper investigates Composition Trees as a formal graphic language to present software processes defined in a VSE Profile; and then comparing it with software processes defined in ISO/IEC 12207 by generating a CCT. The CCT shows the similarities and differences between processes defined in the two standards in a clear, precise and easy to read way. This comparison helps people who make standards to reason and then improve their new standards; it also help software engineering practitioners to understand and implement the VSE Profile in their projects and then eventually adapt additional elements from more comprehensive standards such as ISO/IEC 12207 into their practices. Even though the research is for the Entry Profile of VSE, the method can also be used for other profiles in VSE or other standards.

So far the research is still at its preliminary stage and the research results are very promising. Some possible further research directions include adding more elements such as work products, activities and tasks in the CT models, integrating multiple processes in one $\mathrm{CT}$, and developing suitable software tools to support this approach.

\section{References}

1. Behavior Engineering Web Site, http://www.behaviorengineering.org/

2. Box, G.E.P., (1979). Robustness in the strategy of scientific model building, in Robustness in Statistics, R.L. Launer and G.N. Wilkinson, Editors. Academic Press: New York.

3. Chrissis, M.B., Konrad, M. and Schrumm, S., CMMI for Development®: Guidelines for Process Integration and Product Improvement, 3rd Edition, Addison-Wesley, 2011.

4. Dromey, R.G. (2006). Climbing Over the 'No Silver Bullet' Brick Wall, IEEE Software, Vol. 23, No. 2, pp.118-120.

5. Dromey, R.G., "Formalizing the Transition from Requirements to Design", Mathematical Frameworks for Component Software, Models for Analysis and Syn., 2006, pp. 173-206

6. Dromey, R. G., System Composition: Constructive Support for the Analysis and Design of Large Systems, SETE-2005, Brisbane, Australia, 2005

7. Feiler P.H., Humphrey, W.S. (1992). Software Process Development and Enactment, Software Engineering Institute, Pittsburgh, CMU/SEY-92-TR-04 p 11 
8. Gibson, D., Goldenson, D., and Kost, K., Performance Results of CMMI-Based Process Improvement, Technical Report CMU/SEI-2006-TR-004, 2006

9. ISO/IEC TR 10000-1:1998, Information technology -- Framework and taxonomy of International Standardized Profiles: General principles and documentation framework

10. ISO/IEC 12207:2008, Information technology - Software engineering - Software life cycle processes

11. ISO/IEC 15288:2008, Information technology - System engineering - System life cycle process

12. ISO/IEC TR 24774 (2007). Software and systems engineering -- Life cycle management -Guidelines for process description.

13. ISO/IEC TR 29110-1:2011, Software engineering -- Lifecycle profiles for Very Small Entities (VSEs) -- Part 1: Overview

14. ISO/IEC 29110-2:2011, Software engineering -- Lifecycle profiles for Very Small Entities (VSEs) -- Part 2: Framework and taxonomy

15. ISO/IEC TR 29110-3:2011, Software engineering -- Lifecycle profiles for Very Small Entities (VSEs) -- Part 3: Assessment guide

16. ISO/IEC 29110-4-1:2011, Software engineering -- Lifecycle profiles for Very Small Entities (VSEs) -- Part 4-1: Profile specifications: Generic profile group

17. ISO/IEC TR 29110-5-1-2:2011, Software engineering -- Lifecycle profiles for Very Small Entities (VSEs): Management and engineering guide: Generic profile group: Basic profile

18. Laporte, C.Y., Alexandre, S., Renault, A. Developing International Standards for Very Small Enterprises, Computer, V41(3), pp 98-101, 2008

19. Podorozhny, R. M., Perry, D.E. et al, Artifact-based functional comparison of software processes, 4th IWSPSM May 2003, pp V.29.1-10

20. Scacchi, W. (2001). Process Models in Software Engineering. Encyclopedia of Software Engineering, $2^{\text {nd }}$ Edition, J.J. Marciniak (ed.) John Wiley and Sons, Inc, New York.

21. Sheard, S. A., The frameworks quagmire, a brief look, Proceedings of the 7th Annual International INCOSE, Symposium (INCOSE'97), 1997

22. Tuffley, D., Rout, T., Behavior Engineering as a Process Model Verification Tool, Proceedings of the $10^{\text {th }}$ International SPICE conference, 2010

23. Varkoi, T., Process Assessment in Very Small Entities - An ISO/IEC 29110 based method, $7^{\text {th }}$ QUATIC, 2010.

24. Wen, L., Dromey, R.G., "From Requirements Change to Design Change: A Formal Path", Proceedings of the $2^{\text {nd }}$ IEEE International Conference on SEFM, pp.104-113, 2004.

25. Wen, L., Tuffley, D. \& Rout T., Using Composition Trees to Model and Compare Software Process, SPICE2011, pp 1-15, 2011

26. Wen, L. \& Tuffley, D., Formalizing Manufacturing Process Modeling using Composition Trees, Advanced Materials Research, Vols 399-401(2012) pp 1852-1855, 2012

27. Wynn, D.C., Model-Based Approaches to Support Process Improvement in Complex Product Development, PhD Thesis, University of Cambridge, 2007. 